\title{
A graph theoretic approach to matrix inversion by partitioning *
}

\author{
By
}

\author{
FRANK HARARY
}

\begin{abstract}
Let $M$ be a square matrix whose entries are in some field. Our object is to find a permutation matrix $P$ such that $P M P^{-1}$ is completely reduced, i.e., is partitioned in block triangular form, so that all submatrices below its diagonal are 0 and all diagonal submatrices are square and irreducible. Let $A$ be the binary $(0,1)$ matrix obtained from $M$ by preserving the o's of $M$ and replacing the nonzero entries of $M$ by 1's. Then $A$ may be regarded as the adjacency matrix of a directed graph $D$. Call $D$ strongly connected or strong if any two points of $D$ are mutually reachable by directed paths. A strong component of $D$ is a maximal strong subgraph. The condensation $D^{*}$ of $D$ is that digraph whose points are the strong components of $D$ and whose lines are induced by those of $D$. By known methods, we construct $D^{*}$ from the digraph $D$ whose adjacency matrix $A$ was obtained from the original matrix $M$. Let $A^{*}$ be the adjacency matrix of $D^{*}$. It is easy to show that there exists a permutation matrix $Q$ such that $Q A^{*} Q^{-1}$ is an upper triangular matrix. The determination of an appropriate permutation matrix $P$ from this matrix $Q$ is straightforward.
\end{abstract}

Our object is to develop a procedure to assist in finding the inverse of a given matrix $M=\left[m_{i j}\right]$, by finding a permutation matrix $P$ such that $P M P^{-1}$ is in block triangular form. Standard techniques then apply which enable one to write $M^{-1}$ as a partitioned matrix whose blocks are expressions involving the inverses of the diagonal submatrices of $P M P^{-1}$. Of course, this will only be possible when the original matrix is sufficiently sparse, i.e., contains sufficiently many zeros. In addition, this depends on the strategic location of these zeros.

In recent years, there have been many papers which relate graphs and matrices. Some of these are mentioned in HARARY [1] and others in Parter [6]. The approach to matrix inversion which we will describe in this article constitutes a special case of what HOUSEHOLDER [5] calls the method of modification, which is a closed method for inverting matrices. Forsythe [4] notes that "With any method (of matrix inversion) the partitioning may greatly increase the speed by enabling subsidiary matrix inversions to take place entirely within the high speed memory".

* This was an informal talk at the International Symposium on Matrix Computation sponsored by SIAM and held in Gatlinburg, 'Tennessee, April 24-28, 1961 and was an invited address at the SIAM meeting in Stillwater, Oklahoma on August 31, 1961. 


\section{Preliminaries}

Let $M=\left[m_{i j}\right]$ be any square matrix of order $n$ whose entries are from an arbitrary field. We define the binary matrix (with entries 0 or 1) $A=A(M)=\left[a_{i j}\right]$ by:

$$
a_{i j}=\left\{\begin{array}{l}
0 \text { if } m_{i j}=0 \text { or } i=j \\
1 \text { otherwise. }
\end{array}\right.
$$

We now construct the directed graph or digraph $D=D(M)$ whose adjacency matrix is $A$ by introducing $n$ points $v_{1}, v_{2}, \ldots, v_{n}$ and by drawing a (directed) line from $v_{i}$ to $v_{j}$ whenever $a_{i j}=1$.

For example, if the given matrix (with rational entries) is

$$
M=\left[\begin{array}{rrrrrr}
11 & 0 & -3 & -4 & 11 & 0 \\
0 & -1 & 1 & 0 & 0 & 0 \\
0 & 0 & -1 & 0 & 0 & 1 \\
3 & 5 & -1 & -1 & 2 & 2 \\
3 & 2 & 0 & -1 & 3 & 1 \\
0 & 0 & -2 & 0 & 0 & 3
\end{array}\right]
$$

then

$$
A=\left[\begin{array}{llllll}
0 & 0 & 1 & 1 & 1 & 0 \\
0 & 0 & 1 & 0 & 0 & 0 \\
0 & 0 & 0 & 0 & 0 & 1 \\
1 & 1 & 1 & 0 & 1 & 1 \\
1 & 1 & 0 & 1 & 0 & 1 \\
0 & 0 & 1 & 0 & 0 & 0
\end{array}\right]
$$

so that the digraph of $M$ is

$D:$

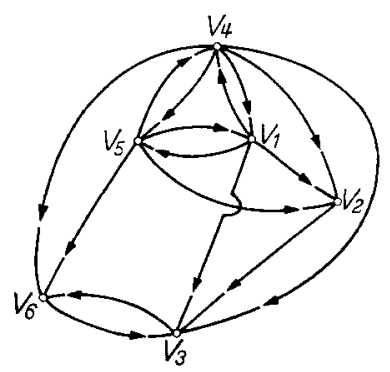

Fig. 1

If $P$ is a permutation matrix, we call $P M P^{-1}$ a permutation of matrix $M$. It is standard terminology in matrix theory to say that a matrix $M$ is decomposable if there exists a permutation of $M$ which has the partitioned form

$$
\left[\begin{array}{cc}
M_{11} & 0 \\
0 & M_{22}
\end{array}\right]
$$


where $M_{11}$ and $M_{22}$ are square submatrices; otherwise $M$ is indecomposable. Similarly if there is a permutation of $M$ which has the partitioned form

$$
\left[\begin{array}{cc}
M_{11} & M_{12} \\
0 & M_{22}
\end{array}\right],
$$

then $M$ is reducible; otherwise $M$ is irreducible. These two properties of a square matrix $M$ are reflected in its digraph $D$.

A digraph is strongly connected or strong if any two points are mutually reachable by means of directed paths. A strong component of $D$ is a maximal strong subgraph. The subgraph of $D$ generated by a set $S$ of points is denoted by $\langle S\rangle$, has $S$ as its set of points, and contains all those lines of $D$ which join two points of $S$. Thus we see that the strong components of the digraph $D$ of Figure 1 are $S_{1}=\left\langle v_{1}, v_{4}, v_{5}\right\rangle, S_{2}=\left\langle v_{2}\right\rangle, S_{3}=\left\langle v_{3}, v_{6}\right\rangle$.

A digraph is weakly connected or weak if and only if the ordinary graph obtained by ignoring the directions of the lines of $D$ is connected. Thus every strong digraph is weak, but not conversly.

The following two statements are implicitly contained in [1] and [2].

Theorem [1]. A square matrix $M$ is irreducible if and only if its digraph $D$ is strongly connected.

Theorem [2]. A square matrix $M$ is indecomposable if and only if its digraph $D$ is weakly connected.

To every digraph $D$, there is associated another digraph $D^{*}$ called its condensation. The points of $D^{*}$ are the strong components $S_{1}, S_{2}, \ldots, S_{t}$ of $D$ and there is a line in $D^{*}$ from $S_{i}$ to $S_{j}$ provided $i \neq j$ and there is at least one line in $D$ from a point of $S_{i}$ to a point of $S_{j}$. In Figure 2 we show the condensation $D^{*}$ of the digraph $D$ of Figure 1 .

$D^{*}$ :

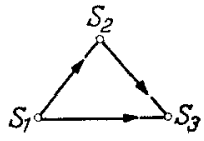

Fig. 2

Let $v$ be any point of $D$. The indegree of $v$, denoted id $v$, is the number of directed lines of $D$ which terminate at $v$; its outdegree, od $v$, is the number of lines of $D$ from point $v$. An isolate of $D$ is a point $v$ such that id $v=\operatorname{od} v=0$; $v$ is a transmitter if id $v=0$ but od $v>0$; and $v$ is a receiver if od $v=0$, id $v>0$.

A (directed) cycle of $D$ is a subgraph consisting of lines of the form $v_{1} v_{2}$, $v_{2} v_{3}, \ldots, v_{k} v_{1}$ where the points $v_{1}, v_{2}, \ldots, v_{k}$ are distinct. A digraph is acyclic if it has no cycles. The following theorem, proved in [3], serves as a lemma for Theorem 1.

Theorem [3]. If a digraph is acyclic, then it has a point of indegree 0 and a point of outdegree 0 .

The next theorem characterizes an acyclic digraph in terms of its adjacency matrix, and will be useful in our algorithm. 
Theorem 1. For any digraph $D$ the following statements are equivalent:

(1) $D$ is acyclic.

(2) $D^{*}=D$, i.e., every point of $D$ is a strong component and conversely.

(3) There exists a permutation of the adjacency matrix $A$ of $D$ in which every entry below the diagonal is 0 .

Proof. The equivalence of the first two conditions is immediate. If $D$ has a cycle, then no one of the points of this cycle can constitute a strong component. On the other hand, any point of $D$ which is not a strong component must lie on a cycle.

We now show the equivalence between conditions (1) and (3). If we are given that the adjacency matrix $A$ (or a permutation thereof) is upper triangular, i.e., has every entry below its diagonal 0 , then it is impossible for $D$ to contain a directed cycle. For the occurrence of a cycle in $D$ is necessarily reflected by the presence of at least one nonzero entry below the diagonal of $A$. Intuitively, such an entry represents the return of a directed path to the starting point.

In order to prove that (1) implies (3) we use Theorem [3] to select from $D$ a point $u_{1}$ of indegree 0 . Form the digraph $D-u_{1}$ by deleting from $D$ the point $u_{1}$ and all lines of $D$ on which $u_{1}$ lies. Since $D$ has no cycles, $D-u_{1}$ is also acyclic. Applying Theorem [3] again, there exists in $D-u_{1}$ a point $u_{2}$ of indegree 0 . Continuing this process we obtain an ordering $u_{1}, u_{2}, \ldots$ of the points of $D$ such that $u_{k}$ is a point of indegree 0 in the digraph $D-\left\{u_{1}, u_{2}, \ldots, u_{k-1}\right\}$. On writing the adjacency matrix of $D$ with regard to this ordering of its points, we see that every entry of the first column below the 1,1 cell is 0 since $u_{1}$ has indegree 0 in $D$, every entry in the second column below the 2,2 cell is 0 since $u_{2}$ is a point of indegree 0 in $D-u_{1}$, etc., completing the proof.

Since $D^{*}$ is acyclic, its adjacency matrix $A^{*}$ can be permuted into upper triangular form. Hence the theorem provides a partial ordering of the strong components of any digraph in accordance with the reachability relation.

The following algorithm shows how to find a permutation matrix $P$ with the property that $P M P^{-1}$ is in block triangular form and every diagonal submatrix is irreducible. Having found an expression for the inverse of $P M P^{-1}$ we may then write

$$
M^{-1}=P^{-1}\left(P M P^{-1}\right)^{-1} P
$$

\section{The algorithm}

1. Take the given square matrix $M=\left[m_{i j}\right]$ and write the corresponding binary matrix $A=\left[a_{i j}\right]$. The matrix $A$ contains all the information needed to draw the digraph $D$, but this is not necessary since it is possible to describe every step in matrix terms.

2 . Let $R=\left[r_{i j}\right]$ be the reachability matrix of the digraph $D$ whose adjacency matrix is $A$. Its entries are defined by:

$$
r_{i j}= \begin{cases}1 & \text { if there is a path in } D \text { from } v_{i} \text { to } v_{j} \\ 0 & \text { otherwise. }\end{cases}
$$

Thus $R$ is the adjacency matrix of the transitive closure of the binary relation represented by $D$. It is shown in [3] that the matrix $R$ is expressible in terms 
of the matrix $A$ by a formula using boolean arithmetic. We denote by $A^{2} \#$ the binary matrix obtained from $A^{2}$ by using the rule that $1+1=1$. In terms of this operation we have the following formula from [3],

$$
R=(A+I)^{n-1} \# .
$$

3. We now find the strong components of $D$ by exploiting the matrix $R$. Let $R \times R^{\prime}$ be the elementwise product of the matrix $R$ with its transpose, so that the $i, j$ entry of $R \times R^{\prime}$ is $r_{i j} r_{j i}$. This of course is a symmetric matrix. The strong component of $D$ containing point $v_{1}$ consists of all those points $v_{j}$ such that the $1, j$ entry of $R \times R^{\prime}$ is 1 . Call this strong component $S_{1}$ and delete the rows and columns corresponding to those points from the matrix $R \times R^{\prime}$, retaining in the resulting submatrix the original numbering of the rows and columns of $R \times R^{\prime}$. Continue this process to find the remaining strong components $S_{2}$, $S_{3}, \ldots, S_{i}$.

Thus far the strong components of $D$ have been identified. These are the points of $D^{*}$. The lines of $D^{*}$ are obtained by referring to the matrix $R$, since there will be a line from $S_{i}$ to $S_{j}$ if and only if $i \neq j$ and if every point of $S_{j}$ is reachable from each point of $S_{i}$.

4. With the information available from the preceding step we may now find the adjacency matrix $A^{*}$ of $D^{*}$, in which the rows and columns are both ordered in accordance with the strong components $S_{1}, S_{2}, \ldots, S_{t}$. Note that the definition of $D^{*}$ guarantees that the diagonal of $A^{*}$ is 0 .

5 . We know by Theorem [3] that $A^{*}$ has at least one column whose sum is 0 . Call the corresponding strong component $S_{i_{1}}$. Delete its row and column and take any column in the resulting submatrix whose sum is 0 . Call its strong component $S_{i_{2}}$, etc. Now write the upper triangular matrix $B^{*}$ obtained from $A^{*}$ by reordering the rows and columns $S_{i_{1}}, S_{i_{2}}, \ldots, S_{i_{t}}$. This is equivalent to writing $B^{*}=Q A^{*} Q^{-1}$ for the appropriate permutation matrix $Q$ whose order is the number of strong components of $D$.

6 . We now relabel the points of $D$ as follows. Let $u_{1}, u_{2}, \ldots, u_{n_{1}}$ be any ordering of the points in the strong component $S_{i_{1}}$. Let $u_{n_{1}+1}, \ldots, u_{n_{1}+n_{\mathrm{a}}}$ be any ordering of the points in $S_{i_{2}}$, etc. Let $P$ be the permutation matrix such that $B=P A P^{-1}$ is the result of changing the ordering of the points of $D$ from $v_{1}, v_{2}, \ldots, v_{n}$ to $u_{1}, u_{2}, \ldots, u_{n}$. Now partition the matrix $B$ into submatrices by drawing horizontal lines after the first $n_{1}$ rows, after $n_{2}$ additional rows, etc., and drawing corresponding vertical lines. Thus this partitioning is in block triangular form.

7. Now apply the same permutation matrix $P$ to the original matrix $M$ to obtain $P M P^{-1}$, and partition it in the same manner. It is also in block triangular form:

$$
P M P^{-1}=\left[\begin{array}{ccccc}
M_{11} & M_{12} & M_{13} & \ldots & M_{1 t} \\
0 & M_{22} & M_{23} & \ldots & M_{2 t} \\
0 & 0 & M_{33} & \ldots & M_{3 t} \\
\ldots & \ldots & \ldots & \ldots & \ldots \\
0 & 0 & 0 & \ldots & M_{t t}
\end{array}\right] .
$$


8 . It is well known that if $M_{11}$ and $M_{22}$ are nonsingular submatrices, then

$$
\left[\begin{array}{cc}
M_{11} & M_{12} \\
0 & M_{22}
\end{array}\right]^{-1}=\left[\begin{array}{cc}
M_{11}^{-1} & -M_{12}^{-1} M_{12} M_{22}^{-1} \\
0 & M_{22}^{-1}
\end{array}\right] .
$$

Thus this algorithm shows how to express the inverse of a given matrix in terms of the inverses of the submatrices $M_{i i}$ corresponding to the strong components of its digraph, by iterating formula (3). Clearly $M$ is nonsingular if and only if every submatrix $M_{i i}$ is nonsingular, since $|M|=\prod\left|M_{i i}\right|$. The following statement is implied by the algorithm.

Theorem 2. For any square matrix $M$ with entries from an arbitrary field, there exists a permutation matrix $P$ such that $P M P^{-1}$ is block upper triangular and completely reduced, and the construction of $P$ is determined by the strong components of the digraph of $M$.

A generalization of formula (3) to obtain the inverse of any partitioned matrix in the following specialized block triangular form appears in [7]:

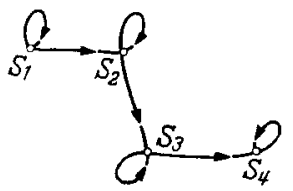

$$
M=\left[\begin{array}{cccc}
M_{11} & M_{12} & 0 & 0 \\
0 & M_{22} & M_{23} & 0 \\
0 & 0 & M_{33} & M_{34} \\
0 & 0 & 0 & M_{44}
\end{array}\right] .
$$

Clearly the condensed digraph corresponding to such a partitioning consists of a single directed path with a loop at every point, as shown. Each strong component of this digraph contains just one point.

\section{An illustration}

Let us see how this algorithm applies to the matrix $M$ whose digraph $D$ is shown in Figure 1. Its condensation $D^{*}$ (Figure 2) shows that the strong components are already numbered so that $S_{1}$ is a transmitter of $D^{*}$ and $S_{2}$ is a transmitter of $D^{*}-S_{1}$. In this case, the strong components are simply ordered. It remains to reorder the points of $D$ within these consecutive strong components. The following table shows the old names of the points $v_{i}$ and their new names $u_{i}$.

Old order $\begin{array}{lllllll}v_{1} & v_{2} & v_{3} & v_{4} & v_{5} & v_{6}\end{array}$

New order $u_{2} \quad u_{4} \quad u_{6} \quad u_{3} \quad u_{1} u_{5}$.

According to this table, the matrix $P$ by which $M$ should be permuted to attain block triangular form is:

$$
P=\left[\begin{array}{llllll}
0 & 0 & 0 & 0 & 1 & 0 \\
1 & 0 & 0 & 0 & 0 & 0 \\
0 & 0 & 0 & 1 & 0 & 0 \\
0 & 1 & 0 & 0 & 0 & 0 \\
0 & 0 & 0 & 0 & 0 & 1 \\
0 & 0 & 1 & 0 & 0 & 0
\end{array}\right] .
$$


The partition of $P M P^{-1}$ using the strong components of $D$ is:

$$
P M P^{-1}=\left[\begin{array}{rrr:r:rr}
3 & 3 & -1 & 2 & 1 & 0 \\
11 & 11 & -4 & 0 & 0 & -3 \\
2 & 3 & -1 & 5 & 2 & -1 \\
\hdashline 0 & 0 & 0 & -1 & 0 & 1 \\
\hdashline 0 & 0 & 0 & 0 & 3 & -2 \\
0 & 0 & 0 & 0 & 1 & -1
\end{array}\right] .
$$

Iterated applications of equation (3) show that the inverse of

is given by

$$
M=\left[\begin{array}{ccc}
M_{11} & M_{12} & M_{13} \\
0 & M_{22} & M_{23} \\
0 & 0 & M_{33}
\end{array}\right]
$$

$$
M^{-1}=\left[\begin{array}{ccc}
M_{11}^{-1} & -M_{11}^{-1} M_{12} M_{22}^{-1} & -M_{11}^{-1} M_{13} M_{33}^{-1}+M_{11}^{-1} M_{12} M_{23}^{-1} M_{23} M_{33}^{-1} \\
0 & M_{22}^{-1} & -M_{22}^{-1} M_{23} M_{33}^{-1} \\
0 & 0 & M_{33}^{-1}
\end{array}\right]
$$

from which

Hence by equation (1),

$$
\left(P M P^{-1}\right)^{-1}=\left[\begin{array}{rrrrrr}
1 & 0 & -1 & -3 & 3 & -8 \\
3 & -1 & 1 & 11 & -18 & 49 \\
11 & -3 & 0 & 22 & -42 & 115 \\
0 & 0 & 0 & -1 & 1 & -3 \\
0 & 0 & 0 & 0 & 1 & -2 \\
0 & 0 & 0 & 0 & 1 & -3
\end{array}\right] .
$$

$$
M^{-1}=\left[\begin{array}{rrrrrr}
-1 & 11 & 49 & 1 & 3 & -18 \\
0 & -1 & -3 & 0 & 0 & 1 \\
0 & 0 & -3 & 0 & 0 & 1 \\
-3 & 22 & 115 & 0 & 11 & -42 \\
0 & -3 & -8 & -1 & 1 & 3 \\
0 & 0 & -2 & 0 & 0 & 1
\end{array}\right] .
$$

Of course the matrix of this illustration can be inverted more quickly by direct methods. In general, the effectiveness of the algorithm increases with the size, complexity, and the number of strong components of the digraph of the given matrix.

If the digraph of the given matrix is already strongly connected, then this algorithm does not help at all. This will be the case very often. For example, if the given matrix contains only positive entries, then its digraph is complete since there is a line from each point to every other point. It is also possible to have a matrix $M$ with many zeros to which this method will not apply. This will be the case for example, when the digraph $D(M)$ contains a cycle passing 
through every point. Thus the digraph is necessarily strong. We see from these two examples that it is not so much a matter of how many zeros a matrix has but rather their strategic location. It would be interesting to find a precise practical procedure to aid in the inversion of an irreducible matrix by exploiting the graphical properties of its strong digraph. In a sense, this is the aim of Kron's "method of tearing".

\section{References}

[1] Harary, F.: A graph theoretic method for the complete reduction of a matrix with a view toward finding its eigenvalues. J. of Mathematics and Physics 38, 104-111 (1959).

[2] -, and I. C. Ross: A description of strengthening and weakening group members. Sociometry 22, 139-147 (1959).

[3] - On the consistency of precedence matrices. J. of the Association of Computing Machinery 7, 255-259 (1960).

[4] Forsythe, G. E.: Solving linear algebraic equations can be interesting. Bulletin of the Americal Mathematical Society 59, 299-329 (1953).

[5] Householder, A. S.: A survey of some closed methods for inverting matrices. SIAM J. 5, 155-169 (1957).

[6] Parter, S.: On the eigenvalues and eigenvectors of a class of matrices. SIAM J. 8, 376-388 (1961).

[7] Swift, G.: A comment on matrix inversion by partition. SIAM Review 2, $132-133$ (1960).

The University of Michigan

Mathematics Department

Ann Arbor, Michigan

(Received August 18, 1961) 